\title{
Reaching millikelvin resolution in Raman distributed temperature sensing using image processing
}

\author{
Marcelo A. Soto*, Jaime A. Ramírez, Luc Thévenaz \\ EPFL Swiss Federal Institute of Technology, Institute of Electrical Engineering \\ SCI STI LT, Station 11, CH-1015 Lausanne, Switzerland \\ *E-mail: marcelo.soto@epfl.ch
}

\begin{abstract}
Image processing is proposed and experimentally demonstrated to improve the capabilities of Raman distributed optical fibre sensors. The here reported technique consists in stacking consecutive one-dimensional Raman Stokes and anti-Stokes traces in two-dimensional data arrays (one for each Raman component), which are then processed by an image denoising algorithm. Owing to the high level of correlation between consecutive measurements in conventional Raman sensing, it is experimentally demonstrated that this newly-proposed two-dimensional denoising approach provides a significant signalto-noise ratio improvement, which in this case reaches $13.6 \mathrm{~dB}$ with no hardware modification to the conventional set-up. Experimental results demonstrate Raman distributed sensing with a remarkably enhanced temperature resolution of $4 \mathrm{mK}$ at $9 \mathrm{~km}$ distance, which is obtained with $2 \mathrm{~m}$ spatial resolution and a short acquisition time of $35 \mathrm{~s}$.
\end{abstract}

Keywords: Optical fibre sensor, Raman scattering, distributed fibre sensor, image processing

\section{INTRODUCTION}

After three decades of research and development, Raman distributed optical fibre sensing ${ }^{1,2}$ is nowadays the most mature and consolidated technology for distributed temperature measurements. The time-domain interrogation approach requires launching high-power optical pulses into a sensing fibre, while the spontaneous Raman anti-Stokes backscattered intensity is measured as a function of the fibre location ${ }^{1,2}$. To compensate for potential laser power fluctuations and some local fibre losses, temperature-dependent Raman anti-Stokes traces are typically normalized by temperature-independent Rayleigh ${ }^{2}$ or Raman Stokes ${ }^{1}$ traces. Following a calibration procedure, a distributed temperature profile can be obtained along several kilometres of optical fibre with a spatial resolution of a few metres. One of the main drawbacks of the method is the low intensity of the spontaneous Raman backscattering, which leads, in principle, to noisy measurements that limit the accuracy of the measured temperature. To improve the measurement precision, Raman sensors typically employ multimode fibres ${ }^{3}$ (MMF), which allow using higher power pulses before activating detrimental nonlinear effects, at the cost of an impaired spatial resolution at long sensing ranges due to modal dispersion. MMFs also enable a higher capture of Raman backscattered power, which translates into traces with higher signal-to-noise ratio (SNR). Although a higher spontaneous Raman backscattered power can reach the detector when using MMFs, traces with limited SNR are typically obtained especially at long sensing ranges, thus constraining the best attainable temperature resolution of the sensor. To improve the precision of the temperature measurements, a large number of traces is typically averaged; however, this generally leads to very long acquisition times, especially if highly-accurate temperature sensing is targeted. To overcome this tradeoff between measurement time and temperature resolution, advanced techniques have been proposed in the last decade for enhancing the SNR and reducing the temperature uncertainty of the measurements. Methods such as optical pulse coding ${ }^{3,4}$, wavelet denoising ${ }^{5}$ and the use of only anti-Stokes traces in a double-ended scheme ${ }^{6}$ have demonstrated to be efficient techniques to improve the capabilities of Raman distributed sensors. In contrast to those one-dimensional (1D) approaches, a two-dimensional (2D) approach based on image processing has been recently proposed to improve the SNR of Brillouin distributed sensors ${ }^{7}$, resulting in a much enhanced performance when compared to traditional 1D approaches. Preliminary results ${ }^{8}$ have confirmed that such a $2 \mathrm{D}$ image processing approach can also be extended to other type of sensors.

In this paper we report on the use of image processing to enhance the performance of Raman distributed fibre sensors. The method here consists in forming two 2D data structures in the spatio-temporal domain by stacking together consecutive 1D traces of the Raman anti-Stokes and Stokes backscattered intensity. Measurement noise is then removed employing 2D image denoising methods. Using a technique so-called non-local means ${ }^{9}$ (NLM), an SNR enhancement of $13.6 \mathrm{~dB}$ is here demonstrated, enabling distributed temperature measurements along a $9 \mathrm{~km}$-long range with a remarkably enhanced resolution of $4 \mathrm{mK}$ and $2 \mathrm{~m}$ spatial resolution, within a measurement time of only $35 \mathrm{~s}$. To the best to our knowledge this represents the first Raman distributed fibre sensor ensuring millikelvin resolution with such a low measurement time. 


\section{DENOISING RAMAN BACKSCATTERING TRACES WITH IMAGE PROCESSING}

\subsection{Denoising one-dimensional time-domain Raman traces with $2 \mathrm{D}$ image denoising}

Raman distributed optical fibre sensors are intensity-based systems ${ }^{1,2}$, and therefore the precision in the obtained temperature profile depends directly on the SNR of the measured Raman anti-Stokes and Stokes (or Rayleigh) traces. The here reported method increases the SNR of time-domain Raman traces, and therefore the temperature accuracy of the sensor, by using a 2D denoising approach based on image processing ${ }^{9,10}$. Digital image processing ${ }^{10}$ refers to techniques that are applied to digital images in order to improve quality or facilitate the extraction of relevant information. The mostcommon techniques include texture analysis, segmentation, noise removal, edge detection, among others.

It has been recently demonstrated that image denoising can be efficiently used to enhance the performance from distributed Brillouin fibre sensors ${ }^{7,8}$, by interpreting each distance-frequency data pair as a pixel of a noisy image. In contrast to such a case, where the measured data is by essence two-dimensional, here 2D image denoising is used to remove noise from 1D Raman traces. Therefore two 2D data structures (one for the anti-Stokes and another for the Stokes component) have to be formed in the distance-time $\left(z, T_{i}\right)$ domain by stacking consecutive traces of a sequential measurement, $T_{i}$ designating the moment of the acquisition of the $i^{\text {th }}$ trace. This way each row of the $2 \mathrm{D}$ matrices represents an independent measurement of the Raman anti-Stokes or Stokes trace as a function of distance. Then the 2D data structures are processed by image denoising techniques ${ }^{9,10}$ to remove white-additive noise, which in this case is essentially dominated by the thermal noise added by the photodetector. It should be noted that the principle of Raman distributed sensing is to measure quasi-static temperature changes, in which the measurand slowly changes when compared to the acquisition time, and therefore consecutive traces are typically highly correlated. Image processing here exploits this high degree of similitude and redundancy (in the time and distance domains) existing in Raman distributed measurements.

\subsection{Non-local means denoising for Raman sensing}

In this paper the method of non-local means ${ }^{9}$ is implemented. This method removes noise by a non-local weighted average of pixels inside a limited section of the image. Unlike traditional filtering, the weighting factors are here obtained based on the similarity between different sections of the image. Hence, similarity windows are defined, corresponding to small 2D image patches that are compared over the image. The degree of similarity is evaluated by calculating the Euclidean distance $^{9}$ among all pixels inside the similarity windows of the image. The window size is directly related to size of the details to be preserved. In order to denoise Raman distributed traces (arranged in a 2D structure in the distance-time domain), the similarity window has to be smaller than the spatial and temporal resolutions of the system. A larger window will certainly lead to loss of spatial and/or temporal information. Unfortunately the 2D matrices resulting from the Raman measurements have a large number of data points, especially for long-range sensing systems, and the processing time can therefore turn out to be extremely long. To reduce processing time, a search window is defined ${ }^{9}$, so that the search for redundancy is limited only to partial sections of the data. Furthermore, the weighing factors also depend on a smoothing control parameter $h$, which is chosen based on the noise standard deviation ${ }^{9}$. Large values of $h$ lead to a strong low-pass filtering effect, potentially removing relevant information from the data.

\section{EXPERIMENTAL SETUP}

Figure 1 shows the experimental setup used to implement highly-accurate temperature sensing based on a conventional Raman distributed fibre sensor. The optical source corresponds to a distributed-feedback laser operating at $1552 \mathrm{~nm}$, which is followed by an electro-optic modulator (EOM) to generate optical pulses of $\sim 10 \mathrm{~ns}$, and an erbium-doped fibre amplifier (EDFA) to boost the peak pulse power up to about $4 \mathrm{~W}$ (at the sensing fibre input). Pulses are launched into a 9 km-long 50/125 $\mu \mathrm{m}$ graded-index multimode fibre

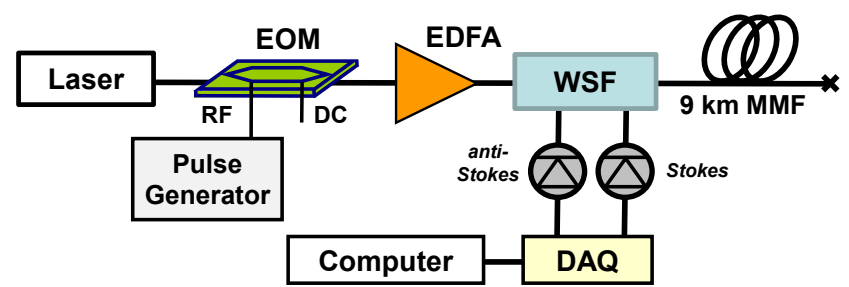

Figure 1. Conventional Raman distributed fibre sensor, using a $9 \mathrm{~km}$-long sensing fibre and $2 \mathrm{~m}$ spatial resolution through a wavelength-selective filter (WSF), which is also used to separate the spontaneous Stokes and anti-Stokes Raman components backscattered from the sensing fibre into two branches. These Raman components are then sent into two parallel $50 \mathrm{MHz}$ avalanche-photodetectors, followed by a data acquisition system (DAQ) connected to a computer. As a result of modal dispersion, the launched optical pulse broadens out with distance, impacting on the spatial resolution. It is experimentally verified that the spatial resolution of the system is $2 \mathrm{~m}$ at $9 \mathrm{~km}$ distance. 


\section{EXPERIMENTAL RESULTS}

Figure 2(a) and 2(b) show the 2D data structures generated by 1D raw data of the Raman anti-Stokes and Stokes traces, respectively (for the sake of clarity only the last $200 \mathrm{~m}$ of fibre are shown). A sampling interval equivalent to $0.5 \mathrm{~m} / \mathrm{pt}$ is used in the acquisition, leading to traces with 18'000 longitudinal points covering the entire $9 \mathrm{~km}$-long sensing range. Each pair of anti-Stokes and Stokes traces is acquired every $35 \mathrm{~s}$; this time was limited in our implementation by the data transfer rate of our equipment, and we believe that this acquisition time could be still reduced. Although the entire data set depicted in Fig. 2 (measured over 50 minutes) could be processed by image denoising, such an approach is only valid for postprocessing all the stored data. However a real-time approach is here followed to evaluate the effectiveness of image processing in a more demanding situation, so that only a limited number of previous traces are used in the processing. For this, small temporal sliding windows of 21 consecutive traces are used to remove noise from the current measurement, taking into account the redundancy of information when it is compared to the 20 previously measured traces. This way, two 2D matrices of $21 \times 18^{\prime} 000$ points are generated; these being updated for each new pair of Raman traces that is measured.
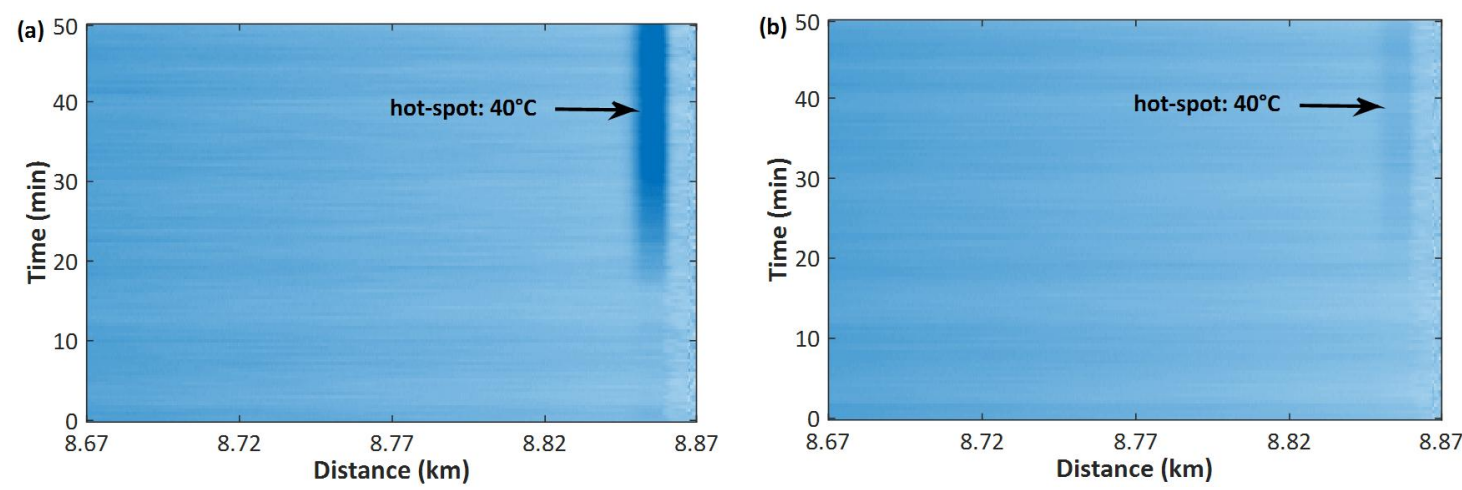

Figure 2. 2D images representing consecutive Raman (a) anti-Stokes and (b) Stokes traces. This corresponds to the entire set of measurements acquired during 50 minutes. After $\sim 15$ minutes of measurements, the temperature of $10 \mathrm{~m}$ of fibre near the farther end of the sensing range was increased from room temperature $\left(25^{\circ} \mathrm{C}\right)$ up to $40^{\circ} \mathrm{C}$.

NLM denoising is performed using a search window of $21 \times 21$, and a similarity window of $3 \times 3$ to ensure a spatial resolution of $2 \mathrm{~m}$ (corresponding to 4 longitudinal points), whilst we set the parameter $h=10 \sigma, \sigma$ being the noise standard deviation. Figure 3(a) shows that the SNR of $33.4 \mathrm{~dB}$ obtained $\sim 9 \mathrm{~km}$ distance with the raw data (averaged $2^{16}$ times) can be remarkably improved up to $47.0 \mathrm{~dB}$ after denoising, representing an SNR enhancement of $13.6 \mathrm{~dB}$. Thus, the temperature resolution of $90 \mathrm{mK}$, obtained from the raw data at the fibre end, can be improved down to $4 \mathrm{mK}$ by NLM, as shown in Fig 3(b). The processing time of each matrix is about $1 \mathrm{~s}$ using a conventional computer with a $3.5 \mathrm{GHz}$ processor and $8 \mathrm{~GB}$ RAM.
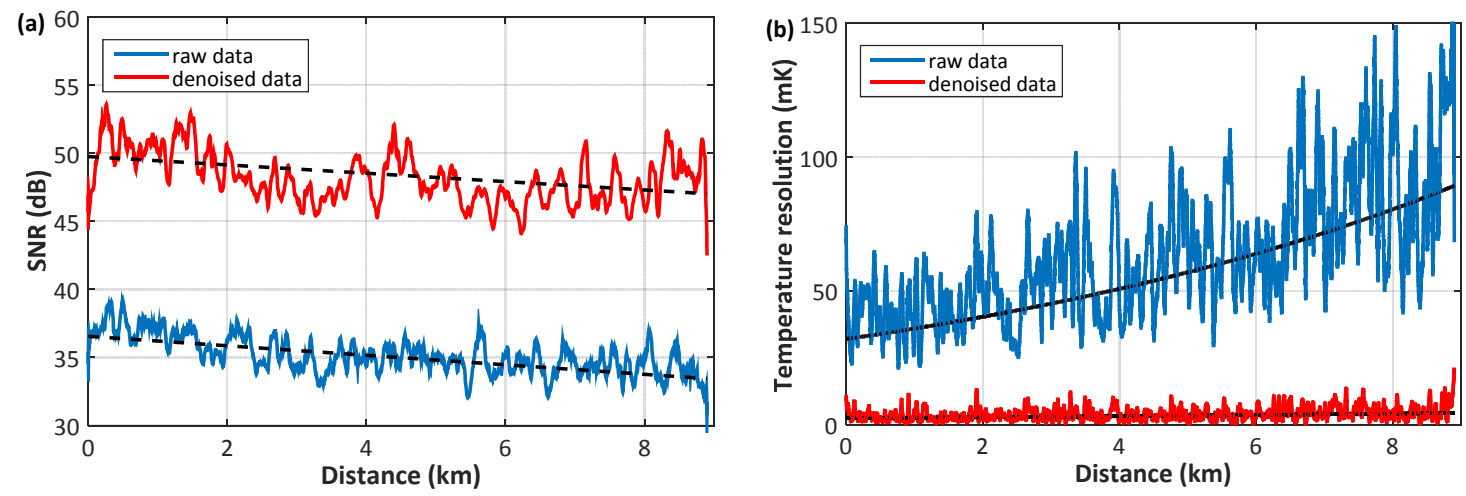

Figure 3. Performance improvement of a $9 \mathrm{~km}$-long Raman distributed sensor employing image processing. (a) SNR of the anti-Stokes trace and (b) temperature resolution vs distance, considering the raw and denoised data using the NLM method. Measurement are obtained with $2^{16}$ averages in $35 \mathrm{~s}$, and $2 \mathrm{~m}$ spatial resolution at a $9 \mathrm{~km}$ distance.

The performance of the 2D NLM denoising has actually been verified for different averaging conditions, ranging from $2^{12}$ up to $2^{20}$ averages. Table 1 summarises the temperature resolution values obtained at $9 \mathrm{~km}$ from the raw and denoised data, as well as the SNR improvement experimentally verified in the anti-Stokes traces. It is possible to observe that the squareroot dependence on the number of averages is actually maintained not only in the raw data, but also in the data denoised 
Table 1. Impact of 2D NLM image denoising on Raman distributed sensors for different numbers of averaged traces.

\begin{tabular}{|c|c|c|c|}
\hline Averages & Temp. resolution (raw) & Temp. resolution (NLM) & SNR improvement \\
\hline $2^{12}=4^{\prime} 096$ & $0.39 \mathrm{~K}$ & $0.016 \mathrm{~K}$ & $13.62 \mathrm{~dB}$ \\
\hline $2^{14}=16^{\prime} 384$ & $0.19 \mathrm{~K}$ & $0.008 \mathrm{~K}$ & $13.58 \mathrm{~dB}$ \\
\hline $2^{16}=65^{\prime} 536$ & $0.09 \mathrm{~K}$ & $0.004 \mathrm{~K}$ & $13.61 \mathrm{~dB}$ \\
\hline $2^{18}=262^{\prime} 144$ & $0.05 \mathrm{~K}$ & - & - \\
\hline $2^{20}=1^{\prime} 048^{\prime} 576$ & $0.026 \mathrm{~K}$ & - & - \\
\hline
\end{tabular}

by the NLM, in which the filtering parameter $h$ has been changed linearly according to the noise standard deviation of each case. An SNR improvement of $\sim 13.6 \mathrm{~dB}$ is demonstrated for the different conditions, up to $2^{16}$ averages, ensuring a reliable temperature resolution of $4 \mathrm{mK}$, as also shown in Fig. 3(b). When the number of averages was increased, no reliable value for the temperature resolution could be obtained, presumably because of thermal fluctuations in the order of $\mathrm{mK}$ affecting the sensing fibre during measurements. It is important to notice that the temperature resolution obtained from the raw data with more than 1 million averages is still worse than the one obtained with only $4 \mathrm{k}$ averages and NLM denoising. This, according to the SNR improvement demonstrated, represents a remarkable 500-fold reduction in the measurement time.

To verify that image processing does not distort the measurements and no relevant information is eliminated, the longitudinal evolution of the measured temperature has been analysed within a $10 \mathrm{~m}$ hot-spot. Figure 4 shows that the applied 2D image denoising has no detrimental impact on the hot-spot measurement, securing a spatial resolution of $2 \mathrm{~m}$ and a correct temperature measurement at the end of the sensing range.

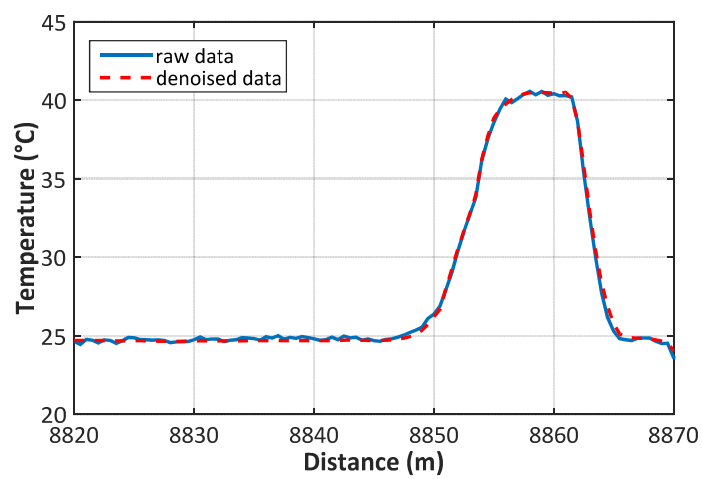

Figure $4.10 \mathrm{~m}$-long hot-spot detection at $\sim 9 \mathrm{~km}$ distance using a spatial resolution of $2 \mathrm{~m}$ and $2^{16}$ averages, for the raw and denoised data.

In conclusion, the use of 2D image processing has been investigated to remove noise from 1D time-domain traces of the Raman anti-Stokes and Stokes backscattered light. This $2 \mathrm{D}$ approach provides a significant reduction in the measurement time, ensuring millikelvin temperature resolutions with acquisition times of a few seconds. We envisage that the 2D denoising technique here reported can be favourably used in Raman distributed sensors operating over standard singlemode fibres, thus overcoming the limitation in the spatial resolution resulting from modal dispersion at long sensing ranges.

The authors acknowledge the support from the Swiss Commission for Technology and Innovation (Project 18337.2 PFNMNM) and are thankful to Omnisens SA for their active discussions and interest in this research.

\section{REFERENCES}

1. Dakin, J. P., Pratt, D. J., Bibby, G. W., Ross, J. N. "Distributed optical fibre Raman temperature sensor using a semiconductor light source and detector," Electron. Lett. 21, 569-570 (1985).

2. Hartog A.H., Leach, A.P., "Distributed temperature sensing in solid-core fibres," Electron. Lett. 21, 1061-1062 (1985).

3. Soto, M. A., Sahu, P. K., Faralli, S., Bolognini, G., Di Pasquale, F., Nebendahl, B., Rueck, C., "Distributed temperature sensor system based on Raman scattering using correlation-codes," Electr. Lett. 43(16), 862-864 (2007).

4. Baronti, F., et al., "SNR enhancement of Raman-based long-range distributed temperature sensors using cyclic Simplex codes," Electr. Lett. 46(17), 1221-1223 (2010).

5. Saxena, M. K., et al. "Raman optical fiber distributed temperature sensor using wavelet transform based simplified signal processing of Raman backscattered signals," Optics \& Laser Technology 65, 14-24 (2015).

6. Soto, M. A, et al., "Impact of Loss Variations on Double-Ended Distributed Temperature Sensors Based on Raman Anti-Stokes Signal Only,” J. Lightwave Technol. 30(8), 1215-1222 (2012).

7. Soto, M. A., Ramírez, J. A., Thévenaz, L. "Intensifying Brillouin distributed fibre sensors using image processing," Proc. SPIE 9634, 24th International Conference on Optical Fibre Sensors, 96342D (September 28, 2015).

8. Soto, M. A., Ramírez, J. A., Thévenaz, L. "Intensifying the response of distributed optical fibre sensors using 2D and 3D image restoration," Nat. Communications (in Press) (2016).

9. Buades, A., Coll, B., Morel, J. M., "A review of image denoising methods, with a new one," Multiscale Model. Simul. 4(2), 490-530 (2005).

10. Russ, J. C., Neal, F. B., “The Image Processing Handbook”. CRC Press 7th Edition (2015). 